\title{
Low frequency stimulation and resulting short-term effects on neuronal activity
}

\author{
Antonio Novellino*, Taina Palosaari and Maurice Whelan
}

\author{
Address: Institute for Health and Consumer Protection, Joint Research Centre, Ispra (VA), 21020, Italy \\ Email: Antonio Novellino* - antonio.novellino@jrc.it \\ * Corresponding author
}

from Eighteenth Annual Computational Neuroscience Meeting: CNS*2009

Berlin, Germany. 18-23 July 2009

Published: 13 July 2009

BMC Neuroscience 2009, I0(Suppl I):P6I doi:I0.I I86/I47I-2202-I0-SI-P6I

This abstract is available from: http://www.biomedcentral.com//47I-2202/I0/SI/P6 I

(C) 2009 Novellino et al; licensee BioMed Central Ltd.

\section{Introduction}

Ex vivo cultured networks of neurons coupled to Micro Electrode Arrays (MEAs) constitute a valuable tool for experimentally investigating changes in neuronal dynamics at different developmental stages and in response to xenobiotic exposure. Spike trains as well as bursts can be easily extracted from background noise and thus it is straightforward to evaluate changes in activity in response to external manipulation. These devices make it possible to record the firing activity of neurons over very long periods of time, even months, and it is possible to follow up the functional development by means of the electrophysiology generated form isolated neurons (i.e. first days) up to fully connected neuronal networks (third week) and that reflects phases of massive overproduction of synaptic connections and subsequent synaptic elimination and stabilization. The longitudinal recordings presented here reveal characteristic changes in firing activity of the developing network.

\section{Materials and methods}

Cryo-preserved cortical neurons from mouse provided by Looza http://www.looza.com were plated onto standard TiN/SiN planar MEA and activity was recorded by means of the Multi Channel System MEA-120 (multichannelsystems.com). The STG2004 delivered the electrical stimulation consisting of 50 biphasic pulses $(250 \mu$ s per phase, 2 v peak-to-peak, duty cycle $50 \%$ ) at $0.2 \mathrm{~Hz}$. Spike trains were extracted according the MC_Rack hard threshold.
Neurons were monitored at least three times per week. A microscope image of the morphology of the network was taken before each recording session. The performed protocol comprised three phases: 1) spontaneous activity recordings, 2) electrical stimulation, 3) control condition recordings $(15+5+5$ minutes in total). The reported results are based on the comparison between the last 5 minutes of the $1^{\text {st }}$ phase and the $3^{\text {rd }}$ phase.

\section{Results}

Spontaneous activity is first recorded at the $2^{\text {nd }}$ Day In Vitro (DIV) at a few sites of the multi-electrode array. From then on, activity is recorded from an increasing number of sites, with firing dynamics changing over time primarily due to the developing network morphology. The low frequency electrical stimulations mainly induced short-term effects; in particular, it switched the neurons to a more active state, while the overnight resting relaxed neuron activity. The described effect is particularly evident in young neurons when the synchronized global behavior is not yet dominant. As the network becomes more mature (second-third week) it is also possible to appreciate an electrophysiological phase-locking effect as previously described [1]. From day to day, individual active sites showed changes in the way they were firing, ranging from low frequency spiking (early days), sporadic sparse bursting, up to regular occurrence of short phases of synchronized network bursting (from the second week) as the most prominent phenomenon [2-5]. 


\section{Conclusion}

The present study shows gradual but significant changes during network development, indicating the sensitivity of the spatio-temporal pattern of firing to structural changes in the network. Results also indicate that the native electrophysiological activity can change significantly when recorded immediately after a low frequency electrical stimulation, that in a short time window tends to boost the native activity. The results suggest that the stable temporal relationships of firing among neurons in network bursts provide optimal conditions for effectuating synaptic plasticity mechanisms, giving spontaneous network bursts an important functional role in enhancing consistency between network connectivity and its dynamic repertoire of firing [4]. If confirmed, our findings provide an innovative method to put young neurons in a super-active state and thus accelerate plasticity mechanisms.

\section{References}

I. Chiappalone M, Vato A, Tedesco MB, Marcoli M, Davide F, Martinoia S: Networks of neurons coupled to microelectrode arrays: a neuronal sensory system for pharmacological applications. Biosens Bioelectron 2003, I 8:627-634.

2. Maeda E, Robinson HPC, Kawana A: The mechanisms of generation and propagation of synchronized bursting in developing networks of cortical neurons. I Neurosci 1995, I 5:6834-6845.

3. Kamioka H, Maeda E, Jimbo Y, Robinson HPC, Kawana A: Spontaneous periodic synchronized bursting during formation of mature patterns of connections in cortical cultures. Neurosci Lett 1996, 206:109-II2.

4. Segev R, Shapira Y, Benvenisto M, Ben-Jacob E: Observations and modeling of synchronized bursting in two-dimensional neural networks. Phys Rev E 200I, 64:I-9.

5. van Pelt J, Vajda I, Wolters PS, Corner MA, Ramakers JGA: Dynamics and plasticity in developing neuronal networks in vitro. Prog Brain Res 2005, 147:173-188.
htp.//www.biomedcentral.com/1471-2202/10/S1/P61 